\title{
Minimal polynomials for Gauss periods with $f=2$
}

\author{
by
}

\section{S. GuraK (San Diego, CA)}

1. Introduction. For an integer $m>1$, fix a primitive $m$ th root of unity $\zeta_{m}=\exp (2 \pi i / m)$ and let $\mathbb{Z}_{m}^{*}$ denote the multiplicative group of reduced residues modulo $m$. Let $H$ be a congruence group of conductor $m$ and of order $f$. It is a classical problem dating back to Gauss [4] to determine the minimal polynomial $f(x)$ of the Gauss periods

$$
\theta_{v}=\sum_{x \in H} \zeta_{m}^{v x} \quad\left(v \in \mathbb{Z}_{m}^{*} / H\right)
$$

corresponding to $H$, or equivalently its reciprocal $F(X)=X^{e} f\left(X^{-1}\right)$ where $e=\phi(m) / f$. (It is known that the $\theta_{v}$ are distinct and $f(x)$ is irreducible over the rational field $\mathbb{Q}$ and that $H$ has conductor $m \equiv 0(\bmod 4)$ if $m$ is even $[6,8]$.)

For $f=1$, the minimal polynomial is the classical cyclotomic polynomial $\psi_{m}(x)$ given by

$$
\psi_{m}(x)=\prod_{d \mid m}\left(1-x^{m / d}\right)^{\mu(d)}=\sum_{k=0}^{\phi(m)} b_{k} x^{k}
$$

which satisfies

$$
\psi_{m}(x)=\frac{\psi_{m / p}\left(x^{p}\right)}{\psi_{m / p}(x)}
$$

for any odd prime $p \mid m$. The polynomial $\psi_{m}(x)$ is self-reciprocal, that is, the coefficients $b_{k}$ satisfy

$$
b_{0}=1, \quad b_{\phi(m)-i}=b_{i} \quad \text { for } 0 \leq i \leq[\phi(m) / 2] .
$$

(Here [ ] denotes the greatest integer function, and $\phi$ and $\mu$ are the usual Euler-phi and Möbius functions, respectively.) 
Gauss himself settled the case $f=2$ when $m=p$ is an odd prime, giving the explicit formula (see [4])

$$
F_{p}(X)=\sum_{r=0}^{e}(-1)^{[r / 2]}\left(\begin{array}{c}
{[e-r / 2]} \\
{[r / 2]}
\end{array}\right) X^{r}
$$

for the reciprocal polynomial for $\zeta_{p}+\zeta_{p}^{-1}$. For $f>2$ it is known $[5,7]$ that no such closed formula exists, but that the beginning coefficients, at least, satisfy a predictable pattern depending polynomially on the distinct prime factors of $m$.

Here I treat the general case $f=2$, showing in Section 2 how to compute the minimal polynomial $F(X)$ for the reciprocals of the Gauss periods (1) when $m$ is composite. This determination is seen to rely on the special cases $H=\{ \pm 1\}$ (and $H=\{1, m / 2-1\}$ when $8 \mid m$ ) of conductor $m$, for which I give a closed formula generalizing (4) for $F(X)$, expressed in terms of the coefficients of the cyclotomic polynomial $\psi_{m^{\prime}}(x)$ in $(2)$, where $m^{\prime}$ is the product of the distinct primes dividing $m$. The details appear in Section 2 . Later in Section 3, I give analogous formulas for quadratic twists of the form $i^{*} \sqrt{l}\left(\zeta_{m}+(-1)^{(l-1) / 2} \zeta_{m}^{-1}\right)$, when $l \mid m^{\prime}$ with $m^{\prime}$ odd and $i^{*}=i^{(l-1)^{2} / 4}$. The latter formulas are expressed in terms of an appropriate Aurifeuille or Schinzel factor $[3,9,13]$ of $\psi_{m^{\prime}}\left((-1)^{(l-1) / 2} x\right)$. Such quadratic twists or integer multiples of them arise classically [12] as values of Kloosterman sums for odd prime powers $p^{\alpha}, \alpha>1$.

2. Minimal polynomials for Gauss periods with $f=2$. My principal aim here is to first give an explicit formula for the minimal polynomial $f(x)$ of the Gauss periods $\theta_{v}$ in (1) when $H=\{ \pm 1\}$ (and for $H=\{1, m / 2-1\}$ when $8 \mid m)$. Then I will show how to employ it to compute $f(x)$ in general when $f=2$. It will be more convenient to express the results in terms of the reciprocal polynomial

$$
F(X)=\prod_{v \in \mathbb{Z}_{m}^{*} / H}\left(1-\theta_{v} X\right)=1+c_{1} X+\cdots+c_{e} X^{e}
$$

where $e=\phi(m) / 2$. Then $\log F(X)=-\sum_{n=1}^{\infty} S_{n} X^{n} / n$ as a formal power series, with $n$th power sums $S_{n}=\sum_{v \in \mathbb{Z}_{m}^{*} / H} \theta_{v}^{n}(n \geq 1)$ satisfying the Newton identities

$$
\begin{array}{rlrl}
S_{r}+c_{1} S_{r-1}+\cdots+c_{r-1} S_{1}+c_{r} r & =0 & & (1 \leq r \leq e) \\
S_{n}+c_{1} S_{n-1}+\cdots+c_{e} S_{n-e}=0 & & (n>e) .
\end{array}
$$

I first consider the case $H=\{ \pm 1\}$ with corresponding Gauss period $\theta_{1}=\zeta_{m}+\zeta_{m}^{-1}$ in (1), and denote its minimal polynomial by $f_{m}(x)$ and corresponding reciprocal polynomial by $F_{m}(X)$. The following result will be 
crucial to the determination of the minimal polynomials here as well as quite useful later in Section 3.

Proposition 1. The reciprocal polynomials

$$
C_{d}(X)=\prod_{v=1, v \neq(d+1) / 2}^{d}\left(1-\left(\zeta_{4 d}^{2 v-1}+\zeta_{4 d}^{-2 v+1}\right) X\right) \quad \text { for } d \geq 1
$$

of degree $2[d / 2]$ are equivalently given by the closed formula

$$
C_{d}(X)=\left(\frac{1+\sqrt{1-4 X^{2}}}{2}\right)^{d}+\left(\frac{1-\sqrt{1-4 X^{2}}}{2}\right)^{d} \quad(d \geq 1)
$$

by the recursion

$$
C_{0}=2, \quad C_{1}(X)=1, \quad C_{d}(X)=C_{d-1}(X)-X^{2} C_{d-2}(X) \quad \text { for } d>1,
$$

by the generating function

$$
\sum_{d=0}^{\infty} C_{d}(X) T^{d}=\frac{2-T}{1-T+X^{2} T^{2}}
$$

by the expansion

$$
C_{d}(X)=\sum_{n=0}^{[d / 2]}(-1)^{n} \frac{d}{d-n}\left(\begin{array}{c}
d-n \\
n
\end{array}\right) X^{2 n}
$$

or the power sums

$$
S_{n}=d\left(\begin{array}{c}
n \\
n / 2
\end{array}\right) \text { or } 0 \quad \text { for } 1 \leq n \leq 2[d / 2]
$$

according as $n$ is even or odd.

Proof. The argument follows that of Gupta and Zagier's in the proof of Theorem 2 in [5], first establishing the equivalence of (8)-(12). With $C_{d}(X)$ defined by (8),

$$
\begin{aligned}
\sum_{d=0}^{\infty} C_{d}(X) T^{d} & =\frac{1}{1+\left(1+\sqrt{1-4 X^{2}}\right) T / 2}+\frac{1}{1-\left(1-\sqrt{1-4 X^{2}}\right) T / 2} \\
& =\frac{2-T}{1-T+X^{2} T^{2}},
\end{aligned}
$$

which gives (10). The recursion (9) follows by multiplying both sides of (10) by $1-T+X^{2} T^{2}$ and then comparing corresponding coefficients of $T^{d}$. The formula (11) follows by expanding the right-hand side of (10) as a geometric series and using the binomial theorem. Specifically,

$$
\frac{2-T}{1-T+X^{2} T^{2}}=(1+(1-T)) \sum_{n=0}^{\infty} \frac{(-1)^{n} T^{2 n} X^{2 n}}{(1-T)^{n-1}}
$$




$$
\begin{aligned}
& =\sum_{n=0}^{\infty} \frac{(-1)^{n} T^{2 n} X^{2 n}}{(1-T)^{n+1}}+\sum_{n=0}^{\infty} \frac{(-1)^{n} T^{2 n} X^{2 n}}{(1-T)^{n}} \\
& =1+\sum_{n=0}^{\infty} T^{n}+\sum_{n=1}^{\infty}(-1)^{n} T^{2 n} X^{2 n}\left(\sum_{j=0}^{\infty}\left(\begin{array}{c}
n+j \\
j
\end{array}\right) T^{j}+\sum_{j=0}^{\infty}\left(\begin{array}{c}
n+j-1 \\
j
\end{array}\right) T^{j}\right) \\
& =1+\sum_{n=0}^{\infty} T^{n}+\sum_{n=1}^{\infty} \sum_{j=0}^{\infty}(-1)^{n}\left\{\left(\begin{array}{c}
n+j \\
j
\end{array}\right)+\left(\begin{array}{c}
n+j-1 \\
j
\end{array}\right)\right\} X^{2 n} T^{2 n+j} \\
& =1+\sum_{n=0}^{\infty} T^{n}+\sum_{n=1}^{\infty} \sum_{j=0}^{\infty}(-1)^{n} \frac{2 n+j}{n+j}\left(\begin{array}{c}
n+j \\
n
\end{array}\right) X^{2 n} T^{2 n+j} \\
& =2+\sum_{d=1}^{\infty} T^{d}\left(\sum_{n=0}^{[d / 2]}(-1)^{n} \frac{d}{d-n}\left(\begin{array}{c}
d-n \\
n
\end{array}\right) X^{2 n}\right) .
\end{aligned}
$$

To establish (12), write $C_{d}(X)$ in (8) as

$$
C_{d}(X)=\left(\frac{1+\sqrt{1-4 X^{2}}}{2}\right)^{d}\left(1+\left(\frac{1-\sqrt{1-4 X^{2}}}{2 X}\right)^{2 d}\right)
$$

Then

$$
\begin{aligned}
\log C_{d}(X)= & d \log \left(\frac{1+\sqrt{1-4 X^{2}}}{2}\right) \\
& -\sum_{\nu=1}^{\infty} \frac{(-1)^{\nu} X^{2 d \nu}}{\nu}\left(\sum_{n=0}^{\infty}\left(\begin{array}{c}
2 n \\
n
\end{array}\right) \frac{X^{2 n}}{n+1}\right)^{2 d \nu}
\end{aligned}
$$

since

$$
A(X)=\frac{1-\sqrt{1-4 X^{2}}}{2 X}=X \cdot \sum_{n=0}^{\infty}\left(\begin{array}{c}
2 n \\
n
\end{array}\right) \frac{X^{2 n}}{n+1}
$$

from the expansion

$$
E(X)=\frac{1+\sqrt{1-4 X^{2}}}{2}=1-\sum_{n=0}^{\infty}\left(\begin{array}{c}
2 n \\
n
\end{array}\right) \frac{X^{2 n+2}}{n+1}
$$

given in [5]. Thus, from (6) and (13) (see also (17)), the power sums

$$
S_{n}=\left\{\begin{array}{ll}
0 & \text { if } n \text { is odd, } \\
d\left(\begin{array}{c}
n \\
n / 2
\end{array}\right) & \text { if } n \text { is even }
\end{array} \quad \text { for } 1 \leq n \leq 2[d / 2]\right.
$$

are sufficient to determine $C_{d}(X)$ from Newton's identities (6). This proves the equivalence of (8)-(12).

It remains to show that $c_{d}(x)=x^{d} C_{d}\left(x^{-1}\right)$ has zeros $2 \cos (\pi \nu / 2 d)$ for $\nu$ odd and $1 \leq \nu \leq 2 d-1$ (this includes the zero $2 \cos (\pi / 2)=0$ when $\nu=d$ 
odd). But from (10), the generating function for the $c_{d}(x)$ is

$$
\sum_{d=0}^{\infty} c_{d}(x) T^{d}=\sum_{d=0}^{\infty} C_{d}\left(x^{-1}\right)(x T)^{d}=\frac{2-x T}{1-x T+T^{2}} .
$$

Substituting $x=z+z^{-1}$ yields

$$
\begin{aligned}
\sum_{d=0}^{\infty} c_{d}\left(z+z^{-1}\right) T^{d} & =\frac{2-\left(z+z^{-1}\right) T}{1-\left(z+z^{-1}\right) T+T^{2}} \\
& =(1-z T)^{-1}+\left(1-z^{-1} T\right)^{-1}=\sum_{d=0}^{\infty}\left(z^{d}+z^{-d}\right) T^{d}
\end{aligned}
$$

Thus $c_{d}\left(z+z^{-1}\right)=0$ iff $z^{d}+z^{-d}=0$ iff $z^{4 d}=1$ with $z^{d}= \pm i$ iff $z=\zeta_{4 d}^{\nu}$ with $\nu$ odd iff $z+z^{-1}=2 \cos (\pi \nu / 2 d)$ for $1 \leq \nu \leq 2 d-1$ with $\nu$ odd. But $c_{d}(x)$ is monic $\left(C_{d}(X)\right.$ has constant term 1$)$ and has degree $d$, so $c_{d}(x)=$ $\prod_{\nu=1, \nu \text { odd }}^{2 d-1}\left(x-\left(\zeta_{4 d}^{\nu}+\zeta_{4 d}^{-\nu}\right)\right)$ is the reciprocal polynomial of $C_{d}(X)$ as defined in (7). This completes the proof of Proposition 1.

Incidentally, the power series $A(X)$ in (14) has an important property that will be useful later.

Lemma 1. For any positive integers $n \geq m$, the coefficient of $X^{n}$ in the expansion $A(X)^{m}$ is $\frac{m}{n}\left(\begin{array}{c}n \\ (n-m) / 2\end{array}\right)$ or 0 according as $n \equiv m(\bmod 2)$ or not.

Proof. The proof proceeds using induction on $m$. With $m=1$, the coefficient of $X^{n}$ is clearly 0 if $n$ is even or

$$
\frac{2}{n+1}\left(\begin{array}{c}
n-1 \\
(n-1) / 2
\end{array}\right)=\frac{1}{n}\left(\begin{array}{c}
n \\
(n-1) / 2
\end{array}\right)
$$

if $n$ is odd. With $m=2, A(X)^{2}=-1+A(X) / X$, so by (14),

$$
A(X)^{2}=-1+\sum_{k=0}^{\infty}\left(\begin{array}{c}
2 k \\
k
\end{array}\right) \frac{X^{2 k}}{k+1} .
$$

It follows that the coefficient of $X^{n}$ is

$$
\frac{2}{n+2}\left(\begin{array}{c}
n \\
n / 2
\end{array}\right)=\frac{2}{n}\left(\begin{array}{c}
n \\
(n-2) / 2
\end{array}\right)
$$

if $n$ even or 0 if $n$ odd. Now assume that the conclusion of the lemma holds for all powers $A(X)^{k}$ up to $k=j$ for some $j \geq 2$, and consider $A(X)^{j+1}=$ $-A(X)^{j-1}+A(X)^{j} / X$ by (16). Thus the coefficient of $X^{n}$ in $A(X)^{j+1}$ is the sum of the coefficient of $X^{n}$ in $-A(X)^{j-1}$ and of the coefficient of $X^{n+1}$ in $A(X)^{j}$. By the induction hypothesis, this sum is 0 if $n \not \equiv j+1(\bmod 2)$ but equals 


$$
-\frac{j-1}{n}\left(\begin{array}{c}
n \\
(n-j+1) / 2
\end{array}\right)+\frac{j}{n+1}\left(\begin{array}{c}
n+1 \\
(n-j+1) / 2
\end{array}\right)=\frac{j+1}{n}\left(\begin{array}{c}
n \\
(n-j-1) / 2
\end{array}\right)
$$

if $n \equiv j+1(\bmod 2)$. This completes the induction so the conclusion of the lemma is proved.

When $m=2^{\alpha}, \alpha>2$, the following result is an immediate consequence of Proposition 1 and the lemma above.

Corollary 1.

$$
F_{2^{\alpha}}(X)=\sum_{n=0}^{2^{\alpha-3}}(-1)^{n} \frac{2^{\alpha-2}}{2^{\alpha-2}-n}\left(\begin{array}{c}
2^{\alpha-2}-n \\
n
\end{array}\right) X^{2 n}
$$

with power sums $S_{n}$ satisfying

$$
S_{n}= \begin{cases}0 & \text { if } n \text { is odd }, \\
2^{\alpha-2}\left(\begin{array}{c}
n \\
n / 2
\end{array}\right)+2^{\alpha-1} \sum_{t=1}^{\left[2^{1-\alpha} n\right]}(-1)^{t}\left(\begin{array}{c}
n \\
\left(n-2^{\alpha-1} t\right) / 2
\end{array}\right) & \text { if } n \text { is even } .\end{cases}
$$

Proof. Clearly $F_{2^{\alpha}}(X)=C_{2^{\alpha-2}}(X)$ by Proposition 1 . Using the expansion

$$
\log \left(\left(1+\sqrt{1-4 X^{2}}\right) / 2\right)=-\sum_{n=1}^{\infty}\left(\begin{array}{c}
2 n \\
n
\end{array}\right) \frac{X^{2 n}}{2 n}
$$

and the lemma above, one obtains the expression for the power sums $S_{n}$ upon comparing coefficients in the expansion of $\log C_{2^{\alpha-2}}(X)$ in (13).

I am now ready to describe $F_{m}(X)$ in general. For $d>0$ put

$$
B_{d}(X)= \begin{cases}\sqrt{1-2 X}\left(V(X)^{d}-W(X)^{d}\right) & \text { if } d \text { is odd } \\ \sqrt{1-4 X^{2}}\left(V(X)^{d}-W(X)^{d}\right) & \text { if } d \text { is even, }\end{cases}
$$

where $V(X)=\frac{1}{2}(\sqrt{1+2 X}+\sqrt{1-2 X})$ and $W(X)=\frac{1}{2}(\sqrt{1+2 X}-\sqrt{1-2 X})$. This sequence has initial terms $B_{1}(X)=1-2 X, B_{2}(X)=1-4 X^{2}, B_{3}(X)=$ $(1-2 X)(1+X), B_{4}(X)=1-4 X^{2}$, and satisfies $B_{n}(X)=B_{n-2}(X)-$ $X^{2} B_{n-4}(X)$ for $n>4$. We have

Proposition 2.

$$
F_{m}(X)=\prod_{d \mid m} B_{m / d}(X)^{\mu(d)} .
$$

Proof. I assert that (i) $B_{d}(X)$ has degree $(d+1) / 2$ with zeros $\left(\zeta_{d}^{\nu}+\right.$ $\left.\zeta_{d}^{-\nu}\right)^{-1}, 0 \leq \nu \leq(d-1) / 2$, if $d$ is odd, (ii) $B_{d}(X)$ has degree $d / 2+1$ with zeros $\left(\zeta_{d}^{\nu}+\zeta_{d}^{-\nu}\right)^{-1}, 0 \leq \nu \leq d / 2$, if $2 \| d$, and (iii) $B_{d}(X)$ has degree $d / 2$ with zeros $\left(\zeta_{d}^{\nu}+\zeta_{d}^{-\nu}\right)^{-1}, 0 \leq \nu \leq d / 2, \nu \neq d / 4$, if $4 \mid d$. Then $B_{m}(X)=\prod_{d \mid m} F_{d}(X)$, since the right side has constant term 1 and accounts for all zeros that are reciprocals of the non-zero values $\zeta_{m}^{\nu}+\zeta_{m}^{-\nu}$ with $0 \leq \nu \leq[m / 2]$ exactly once. Now the statement of the proposition readily follows by Möbius inversion. 
But (i) is essentially Theorem 3 in [5] taking into account the extra factor $1-2 X$ for $\nu=0$. So it remains to establish (ii) and (iii) of the claim. Now if $2 \| d$, say $d=2 d^{\prime}$ with $d^{\prime}$ odd, then $B_{d}(X)=B_{d^{\prime}}(X) B_{d^{\prime}}(-X)$. Thus by (i), $B_{d}(X)$ has distinct zeros $\left(\zeta_{d^{\prime}}^{\nu}+\zeta_{d^{\prime}}^{-\nu}\right)^{-1}=\left(\zeta_{d}^{2 \nu}+\zeta_{d}^{-2 \nu}\right)^{-1}$ and $-\left(\zeta_{d^{\prime}}^{\nu}+\zeta_{d^{\prime}}^{-\nu}\right)^{-1}=\left(\zeta_{d}^{2 \nu+d^{\prime}}+\zeta_{d}^{-2 \nu-d^{\prime}}\right)^{-1}$ for $0 \leq \nu \leq\left(d^{\prime}-1\right) / 2$, or equivalently zeros $\left(\zeta_{d}^{\nu}+\zeta_{d}^{-\nu}\right)^{-1}$ for $0 \leq \nu \leq d^{\prime}=d / 2$, establishing assertion (ii). To settle claim (iii) first note if $4 \| d$, say with $d=4 d^{\prime}$ where $d^{\prime}$ is odd, then $B_{d}(X)=B_{2 d^{\prime}}(X) C_{d^{\prime}}(X)$ with $C_{d^{\prime}}(X)$ as in (7). In this case $B_{d}(X)$ has zeros $\left(\zeta_{d}^{2 \nu-1}+\zeta_{d}^{-2 \nu+1}\right)^{-1}$ for $1 \leq \nu \leq d^{\prime}=d / 4, \nu \neq(d+4) / 8$ from Proposition 1 , and zeros $\left(\zeta_{d}^{2 \nu}+\zeta_{d}^{-2 \nu}\right)^{-1}$ for $0 \leq \nu \leq d^{\prime}=d / 4$ from the above. Restated, $B_{d}(X)$ has distinct zeros $\left(\zeta_{d}^{\nu}+\zeta_{d}^{-\nu}\right)^{-1}$ for $0 \leq \nu \leq d / 2, \nu \neq d / 4$ if $4 \| d$. Arguing similarly using Proposition 1 and the above statement, one obtains (iii) in general when $8 \mid d$ by an induction involving the exact power of 2 dividing $d$. The proof of the proposition is now complete.

I should remark that the statement of Proposition 2 is not new, and was first noted by Watkins and Zeitlin [16] in reciprocal form using the properties of the Chebyshev polynomials $T_{m}(x)$, which are defined by

$$
T_{m}(\cos \theta)=\cos (m \theta)
$$

for positive integers $m$ and all real $\theta$. Indeed, defining

$$
b_{m}(x)=2\left(T_{[m / 2]+1}(x / 2)-T_{[(m-1) / 2]}(x / 2)\right)
$$

they essentially show $b_{m}(x)$ has zeros $2 \cos (2 \pi v / m)$ for $0 \leq v \leq[m / 2]$. Here $B_{m}(X)=X^{[m / 2]+1} b_{m}\left(X^{-1}\right)$.

I now give the main result of this section.

Theorem 1. For $m \neq 2^{\alpha}$,

$$
F_{m}(X)=b_{\phi\left(m^{\prime}\right) / 2} X^{\phi(m) / 2}+\sum_{j=0}^{\phi\left(m^{\prime}\right) / 2-1} b_{j} X^{m j / m^{\prime}} C_{\frac{m}{m^{\prime}}\left(\phi\left(m^{\prime}\right) / 2-j\right)}(X)
$$

where the $b_{j}$ are the coefficients for $\psi_{m^{\prime}}(x)$ given in (2) and the polynomials $C_{d}(X)$ are as in (11).

The power sums $S_{n}$ satisfy

$$
S_{n}=\left\{\begin{array}{l}
\sum_{d \mid m} \mu(d) \frac{m}{d} \sum_{t=1, m t / d \text { odd }}^{[n d / m]}\left(\begin{array}{c}
n \\
(n-m t / d) / 2
\end{array}\right) \quad \text { if } n \text { is odd }, \\
\frac{\phi(m)}{2}\left(\begin{array}{c}
n \\
n / 2
\end{array}\right)+\sum_{d \mid m} \mu(d) \frac{m}{d} \sum_{t=1, m t / d \text { even }}^{[n d / m]}\left(\begin{array}{c}
n \\
(n-m t / d) / 2
\end{array}\right) \\
\text { if } n \text { is even. }
\end{array}\right.
$$


The coefficients $c_{r}$ of $F_{m}(X)$ are given for $1 \leq r<\phi(m) / 2$ by

$$
\begin{aligned}
c_{r}= & \sum_{j=0, j m / m^{\prime} \equiv r(\bmod 2)}^{\left[m^{\prime} r / m\right]}(-1)^{t_{j}} b_{j} \\
& \times \frac{\frac{m}{m^{\prime}}\left(\frac{\phi\left(m^{\prime}\right)}{2}-j\right)}{\frac{m}{m^{\prime}}\left(\frac{\phi\left(m^{\prime}\right)}{2}-j\right)-t_{j}}\left(\begin{array}{c}
\frac{m}{m^{\prime}}\left(\frac{\phi\left(m^{\prime}\right)}{2}-j\right)-t_{j} \\
t_{j}
\end{array}\right)
\end{aligned}
$$

and

$$
c_{\phi(m) / 2}= \begin{cases}\left(\frac{-2}{p}\right) & \text { if } m^{\prime}=p \text { an odd prime }, \\ 1 & \text { otherwise }\end{cases}
$$

where $t_{j}=\left(r-j m / m^{\prime}\right) / 2$.

Proof. I first note that if $F_{m}(X)$ is expressed in terms of the coefficients of $\psi_{m^{\prime}}(x)$ and the polynomials $C_{d}(X)$ as given in the initial statement of the theorem, then formula (19) for the coefficients $c_{r}$ is deduced in routine fashion upon collecting like powers of $X$. The value of $c_{\phi(m) / 2}$ is seen to be

$$
b_{\phi\left(m^{\prime}\right) / 2}+2 \sum_{j=0}^{\phi\left(m^{\prime}\right) / 2-1} b_{j}=\sum_{j=0}^{\phi\left(m^{\prime}\right)} b_{j}=\psi_{m^{\prime}}(1)=1
$$

if $m^{\prime}$ is even (and hence composite since $m \neq 2^{\alpha}$ ), or

$$
b_{\phi\left(m^{\prime}\right) / 2}+(-1)^{\left[\left(\phi\left(m^{\prime}\right)+2\right) / 4\right]} \sum_{j=0}^{\phi\left(m^{\prime}\right) / 2-2}(-1)^{[(j+1) / 2]} 2 b_{j}
$$

if $m^{\prime}$ is odd. The latter expression is

$$
(-1)^{\phi\left(m^{\prime}\right) / 4} \sum_{j=0}^{\phi\left(m^{\prime}\right) / 2-1}(-1)^{j} b_{2 j}=(-1)^{\phi\left(m^{\prime}\right) / 4}\left(\psi_{m^{\prime}}(i)+\psi_{m^{\prime}}(-i)\right) / 2
$$

if $4 \mid \phi\left(m^{\prime}\right)$, or

$$
(-1)^{(p-3) / 4} \sum_{j=0}^{\phi(p) / 2-2}(-1)^{j} b_{2 j+1}=(-1)^{(p-3) / 4}\left(\psi_{p}(i)-\psi_{p}(-i)\right) / 2 i
$$

if $m^{\prime}=p \equiv 3(\bmod 4)$ a prime. Noting that for odd primes $p$,

$$
\psi_{p}(i)= \begin{cases}1 & \text { if } p \equiv 1(\bmod 4), \\ i & \text { if } p \equiv 3(\bmod 4),\end{cases}
$$

and using (3), one finds $\psi_{m^{\prime}}(i)=(-1)^{\phi\left(m^{\prime}\right) / 4}$ whenever $m^{\prime}$ is odd and composite. It now follows readily for $m \neq 2^{\alpha}$ that $c_{\phi(m) / 2}$ is $\left(\frac{-2}{p}\right)$ if $m^{\prime}=p$, an odd prime, and 1 otherwise. 
Now I assert that

$$
F_{m}(X)=E(X)^{\phi(m) / 2} \prod_{d \mid m}\left(1-A(X)^{m / d}\right)^{\mu(d)} .
$$

Then

$$
\begin{aligned}
\log F_{m}(X) & =\frac{\phi(m)}{2} \log E(X)+\sum_{d \mid m} \mu(d) \log \left(1-A(X)^{m / d}\right) \\
& =-\frac{\phi(m)}{2} \sum_{n=1}^{\infty}\left(\begin{array}{c}
2 n \\
n
\end{array}\right) \frac{X^{2 n}}{2 n}-\sum_{d \mid m} \mu(d) \sum_{v=1}^{\infty} \frac{A(X)^{m v / d}}{v}
\end{aligned}
$$

again by using the formal Taylor series for $\log (1-T)$ about $T=0$. By Lemma 1 the coefficient of $X^{n}$ in $\sum_{v=1}^{\infty} A(X)^{m v / d} / v$ is

$$
\sum_{t=1, m t / d \equiv n(\bmod 2)}^{[n d / m]} \frac{m}{d n}\left(\begin{array}{c}
n \\
(n-m t / d) / 2
\end{array}\right)
$$

and so the statements about the power sums $S_{n}$ in the theorem would follow.

In addition, if (20) holds then

$$
\begin{aligned}
F_{m}(X)= & E(X)^{\phi(m) / 2} \psi_{m^{\prime}}\left(A(X)^{m / m^{\prime}}\right) \\
= & \left(E(X)^{m / m^{\prime}}\right)^{\phi\left(m^{\prime}\right) / 2} \sum_{j=0}^{\phi\left(m^{\prime}\right)} b_{j} A(X)^{m j / m^{\prime}} \\
= & b_{\phi\left(m^{\prime}\right) / 2} X^{\phi(m) / 2}+\sum_{j=0}^{\phi\left(m^{\prime}\right) / 2-1} b_{j} X^{m j / m^{\prime}} E(X)^{\frac{m}{m^{\prime}}}\left(\phi\left(m^{\prime}\right) / 2-j\right) \\
& +\sum_{j=0}^{\phi\left(m^{\prime}\right) / 2-1} b_{\phi\left(m^{\prime}\right)-j} X^{m \phi\left(m^{\prime}\right) / 2 m^{\prime}} A(X)^{\frac{m}{m^{\prime}}\left(\phi\left(m^{\prime}\right) / 2-j\right)},
\end{aligned}
$$

since $E(X) A(X)=X$, or

$b_{\phi\left(m^{\prime}\right) / 2} X^{\phi(m) / 2}+\sum_{j=0}^{\phi\left(m^{\prime}\right) / 2-1} b_{j} X^{m j / m^{\prime}}\left(E(X)^{\frac{m}{m^{\prime}}\left(\phi\left(m^{\prime}\right) / 2-j\right)}+\bar{E}(X)^{\frac{m}{m^{\prime}}\left(\phi\left(m^{\prime}\right) / 2-j\right)}\right)$

where $\bar{E}(X)=\left(1-\sqrt{1-4 X^{2}}\right) / 2$, since $\psi_{m^{\prime}}(x)$ is self-reciprocal and $X A(X)$ $=\bar{E}(X)$. But $E(X)^{d}+\bar{E}(X)^{d}$ is just the polynomial $C_{d}(X)$ in Proposition 1 , so the expression for $F_{m}(X)$ in the theorem would follow.

It remains to prove assertion (20). If $m$ is odd then from Proposition 3,

$$
F_{m}(X)=\prod_{d \mid m}\left(\sqrt{1-2 X}\left(V(X)^{m / d}-W(X)^{m / d}\right)\right)^{\mu(d)}
$$




$$
\begin{aligned}
& =V(X)^{\phi(m)} \prod_{d \mid m}\left(1-A(X)^{m / d}\right)^{\mu(d)} \\
& =E(X)^{\phi(m) / 2} \prod_{d \mid m}\left(1-A(X)^{m / d}\right)^{\mu(d)}
\end{aligned}
$$

as asserted, since $A(X)=(\sqrt{1+2 X}-\sqrt{1-2 X}) /(\sqrt{1+2 X}+\sqrt{1-2 X})$. For even $m$ we have $4 \mid m$, so from Proposition 3 ,

$$
F_{m}(X)=\prod_{d \mid m}\left(\sqrt{1-4 X^{2}}\left(V(X)^{m / d}-W(X)^{m / d}\right)\right)^{\mu(d)}
$$

again equaling $E(X)^{\phi(m) / 2} \prod_{d \mid m}\left(1-A(X)^{m / d}\right)^{\mu(d)}$. Thus the assertion (20) is verified so the proof of the theorem is now complete.

I wish to remark that direct calculation of the power sums using the binomial theorem

$$
\left(\zeta_{m}+\zeta_{m}^{-1}\right)^{n}= \begin{cases}\left(\begin{array}{c}
n \\
n / 2
\end{array}\right)+\sum_{j=0}^{n / 2-1}\left(\begin{array}{c}
n \\
j
\end{array}\right)\left(\zeta_{m}^{n-2 j}+\zeta_{m}^{2 j-n}\right) & \text { if } n \text { is even } \\
\sum_{j=0}^{(n-1) / 2}\left(\begin{array}{c}
n \\
j
\end{array}\right)\left(\zeta_{m}^{n-2 j}+\zeta_{m}^{2 j-n}\right) & \text { if } n \text { is odd }\end{cases}
$$

and the fact that the trace (see equation (16) in [3]) satisfies

$$
\operatorname{Tr}_{K / \mathbb{Q}}\left(\zeta_{m}^{v}+\zeta_{m}^{-v}\right)=\sum_{x \in \mathbb{Z}_{m}^{*}} \zeta_{m}^{v x}=\mu(d) \frac{\phi(m)}{\phi(d)}
$$

if $(v, m)=m / d$, where $K=\mathbb{Q}\left(\zeta_{m}+\zeta_{m}^{-1}\right)$, yield a variant form for the $S_{n}$ in (18). Namely,

$$
S_{n}=\left\{\begin{array}{l}
\sum_{d \mid m} \mu(d) \frac{\phi(m)}{\phi(d)} \sum_{t=1,(t, d)=1, m t / d \text { odd }}^{[n d / m]}\left(\begin{array}{c}
n \\
(n-m t / d) / 2
\end{array}\right) \\
\frac{\phi(m)}{2}\left(\begin{array}{c}
n \\
n / 2
\end{array}\right)+\sum_{d \mid m} \mu(d) \frac{\phi(m)}{\phi(d)} \sum_{t=1, m t / d \text { is oven }}^{[n d / m]}\left(\begin{array}{c}
n \\
(n-m t / d) / 2
\end{array}\right) \\
\text { if } n \text { is even. }
\end{array}\right.
$$

However, these are seen to be equivalent using the alternative expression $\psi_{m^{\prime}}(x)=\prod_{v \in \mathbb{Z}_{m^{\prime}}}\left(1-\zeta_{m^{\prime}}^{v} x\right)$ to evaluate $\psi_{m^{\prime}}\left(A^{m / m^{\prime}}\right)$ in (20) before taking logarithms.

Here are a couple of examples to illustrate Theorem 1.

ExAmple 1. Consider $\theta_{1}=\zeta_{27}+\zeta_{27}^{-1}$ in (1). Here $m=27, m^{\prime}=3$ and $\psi_{3}(x)=1+x+x^{2}$ in $(2)$. Direct calculation of the power sums $S_{n}$ yields 
$S_{1}=S_{3}=S_{5}=S_{7}=0, S_{9}=-9, S_{2}=18, S_{4}=54, S_{6}=180$ and $S_{8}=630$ with $F_{27}(X)=1-9 X^{2}+27 X^{4}-30 X^{6}+9 X^{8}+X^{9}$ in agreement with the formulas in Theorem 1 .

EXAmple 2. Now consider $\theta_{1}=\zeta_{15}+\zeta_{15}^{-1}$ in (1). Here $m=m^{\prime}=15$ and $\psi_{15}(x)=1-x+x^{3}-x^{4}+x^{5}-x^{7}+x^{8}$ in (2). Direct calculation of the power sums $S_{n}$ yields $S_{1}=1, S_{2}=9, S_{3}=1, S_{4}=29$ with $F_{15}(X)=$ $1-X-4 X^{2}+4 X^{3}+X^{4}$ again in agreement with Theorem 1 .

The case $m=p^{\alpha}, p$ an odd prime, warrants special consideration.

Corollary 2. For an odd prime $p$,

$$
F_{p^{\alpha}}(X)=X^{\phi\left(p^{\alpha}\right) / 2}+\sum_{j=0}^{(p-3) / 2} X^{p^{\alpha-1} j} C_{p^{\alpha-1}(p-1-2 j) / 2}(X)
$$

with nth power sums $S_{n}$ equal to

$$
p^{\alpha} \sum_{t=1, \text { todd }}^{\left[n p^{-\alpha}\right]}\left(\begin{array}{c}
n \\
\left(n-p^{\alpha} t\right) / 2
\end{array}\right)-p^{\alpha-1} \sum_{t=1, \text { odd }}^{\left[n p^{1-\alpha}\right]}\left(\begin{array}{c}
n \\
\left(n-p^{\alpha-1} t\right) / 2
\end{array}\right)
$$

if $n$ is odd, or

$$
\frac{\phi\left(p^{\alpha}\right)}{2}\left(\begin{array}{c}
n \\
n / 2
\end{array}\right)+p^{\alpha} \sum_{t=1}^{\left[n p^{-\alpha} / 2\right]}\left(\begin{array}{c}
n \\
n / 2-p^{\alpha} t
\end{array}\right)-p^{\alpha-1} \sum_{t=1}^{\left[n p^{1-\alpha} / 2\right]}\left(\begin{array}{c}
n \\
n / 2-p^{\alpha-1} t
\end{array}\right)
$$

if $n$ is even. The coefficients $c_{r}$ of $F_{p^{\alpha}}(X)$ are given for $1 \leq r<\phi\left(p^{\alpha}\right) / 2$ by

$$
c_{r}=\sum_{j=0, j \equiv r(\bmod 2)}^{\left[r p^{1-\alpha}\right]}(-1)^{t_{j}} \frac{p^{\alpha-1}\left(\frac{p-1}{2}-j\right)}{p^{\alpha-1}\left(\frac{p-1}{2}-j\right)-t_{j}}\left(\begin{array}{c}
p^{\alpha-1}\left(\frac{p-1}{2}-j\right)-t_{j} \\
t_{j}
\end{array}\right)
$$

with $c_{\phi\left(p^{\alpha}\right) / 2}=\left(\frac{-2}{p}\right)$, where $t_{j}=\left(r-p^{\alpha-1} j\right) / 2$.

I remark that for $m=p$, the above formula for the coefficients $c_{r}$ reduces to that found by Gauss in (4), in view of the combinatorial identity

$$
\sum_{t=0}^{[r / 2]}(-1)^{t} \frac{\frac{p-1}{2}-(r-2 t)}{\frac{p-1}{2}-(r-t)}\left(\begin{array}{c}
\frac{p-1}{2}-(r-t) \\
t
\end{array}\right)=(-1)^{[r / 2]}\left(\begin{array}{c}
{[(p-1-r) / 2]} \\
{[r / 2]}
\end{array}\right)
$$

for $0 \leq r<(p-1) / 2$. This identity follows readily from the fact that

$$
\begin{aligned}
& \sum_{t=0}^{k}(-1)^{t} \frac{x-2 k+2 t}{x-2 k+t}\left(\begin{array}{c}
x-2 k+t \\
t
\end{array}\right) \\
& =\sum_{t=0}^{k}(-1)^{t}\left(\begin{array}{c}
x-2 k+t \\
t
\end{array}\right)+\sum_{t=1}^{k}(-1)^{t}\left(\begin{array}{c}
x-2 k+t-1 \\
t-1
\end{array}\right)
\end{aligned}
$$




$$
\begin{aligned}
& =\sum_{t=0}^{k}(-1)^{t}\left(\begin{array}{c}
x-2 k+t \\
t
\end{array}\right)-\sum_{t=0}^{k-1}(-1)^{t}\left(\begin{array}{c}
x-2 k+t \\
t
\end{array}\right) \\
& =(-1)^{k}\left(\begin{array}{c}
x-k \\
k
\end{array}\right)
\end{aligned}
$$

for $x>k$.

Next I consider the alternative situation when $H=\{1, m / 2-1\}$ with $8 \mid m$, and denote $F(X)$ in $(5)$ by $G_{m}(X)$. Now one has $\theta_{1}=\zeta_{m}-\zeta_{m}^{-1}=$ $i\left(\zeta_{m}^{m / 4-1}+\zeta_{m}^{1-m / 4}\right)$ in $(1)$, so that $G_{m}(X)=F_{m}(i X)$ with corresponding sums $S_{n}^{-}=0$ if $n$ is odd and $S_{2 n}^{-}=(-1)^{n} S_{2 n}$. The next result now follows immediately from Theorem 1 and Corollary 1.

THEOREM 2. Let $8 \mid m$ and $H=\{1, m / 2-1\}$. The minimal polynomial for the reciprocals of the Gauss periods $\theta_{v}=\zeta_{m}^{v}-\zeta_{m}^{-v}\left(v \in \mathbb{Z}_{m}^{*} / H\right)$ is

$$
\begin{aligned}
G_{m}(X)= & (-1)^{\phi(m) / 4} b_{\phi\left(m^{\prime}\right) / 2} X^{\phi(m) / 2} \\
& +\sum_{j=0}^{\phi\left(m^{\prime}\right) / 2-1} b_{j} X^{m j / m^{\prime}} C_{\frac{m}{m^{\prime}}\left(\phi\left(m^{\prime}\right) / 2-j\right)}(i X)
\end{aligned}
$$

when $m \neq 2^{\alpha}$, with corresponding sums $S_{n}^{-}=0$ if $n$ is odd, and

$$
S_{n}^{-}=(-1)^{n / 2} \frac{\phi(m)}{2}\left(\begin{array}{c}
n \\
n / 2
\end{array}\right)+(-1)^{n / 2} \sum_{d \mid m} \mu(d) \frac{m}{d} \sum_{t=1}^{[n d / m]}\left(\begin{array}{c}
n \\
(n-m t / d) / 2
\end{array}\right)
$$

if $n$ is even. The coefficients of $G_{m}(X)$ are given for $1 \leq r<\phi(m) / 2$ by

$$
c_{r}=\left\{\begin{array}{c}
(-1)^{[r / 2]} \sum_{j=0}^{\left[m^{\prime} r / m\right]}(-1)^{t_{j}} b_{j} \frac{\frac{m}{m^{\prime}}\left(\frac{\phi\left(m^{\prime}\right)}{2}-j\right)}{\frac{m}{m^{\prime}}\left(\frac{\phi\left(m^{\prime}\right)}{2}-j\right)-t_{j}}\left(\begin{array}{c}
\frac{m}{m^{\prime}}\left(\frac{\phi\left(m^{\prime}\right)}{2}-j\right)-t_{j} \\
t_{j}
\end{array}\right),
\end{array}\right.
$$

according as $r$ is even or odd, respectively, with $c_{\phi(m) / 2}=1$. If $m=2^{\alpha}$ $(\alpha>2)$, then

$$
G_{2^{\alpha}}(X)=\sum_{n=0}^{2^{\alpha-3}} \frac{2^{\alpha-2}}{2^{\alpha-2}-n}\left(\begin{array}{c}
2^{\alpha-2}-n \\
n
\end{array}\right) X^{2^{\alpha-2}-2 n}
$$

with corresponding sums $S_{n}^{-}=0$ if $n$ is odd, and

$$
S_{n}^{-}=(-1)^{n / 2} 2^{\alpha-2}\left(\begin{array}{c}
n \\
n / 2
\end{array}\right)+2^{\alpha-1} \sum_{t=1}^{\left[n 2^{1-\alpha}\right]}(-1)^{t+n / 2}\left(\begin{array}{c}
n \\
\left(n-2^{\alpha-1} t\right) / 2
\end{array}\right)
$$

if $n$ is even.

Here is an example to illustrate Theorem 2. 
EXAmple 3. Consider $\theta_{1}=\zeta_{40}+\zeta_{40}^{19}=\zeta_{40}-\zeta_{40}^{-1}$ in (1) where $H=\{1,19\}$ modulo 40. Here $m=40$ and $m^{\prime}=10$ with

$$
\psi_{10}(x)=1-x+x^{2}-x^{3}+x^{4}
$$

in (2). Direct calculation of the power sums $S_{n}$ yields $S_{1}=S_{3}=S_{5}=S_{7}=0$ and $S_{2}=-16, S_{4}=52, S_{6}=-184, S_{8}=668$ with $G_{40}(X)=1+8 X^{2}+$ $19 X^{4}+12 X^{6}+X^{8}$ in agreement with the formulas in Theorem 2 .

Now I return to the general problem to compute the minimal polynomial $F(X)$ for the reciprocals of the Gauss periods (1) for a given congruence group $H$ of conductor $m$ and order $f=2$. This determination is seen to rely on the special cases $H=\{ \pm 1\}$ and $H=\{1, m / 2-1\}$ already discussed. For this purpose some familiarity with congruence groups is needed. (The reader may find the discussion in Section 5 of [6] helpful here.)

Given a congruence group $H$ of conductor $m$ and a positive divisor $d \mid m$, let $H_{d}$ denote the congruence group defined modulo $d$ determined by

$$
H_{d}=\left\{x \in \mathbb{Z} \mid x \equiv x^{\prime}(\bmod d) \text { for some } x^{\prime} \in H\right\} .
$$

If $p^{\alpha} \| m$, where $p$ is prime, then $H_{p^{\alpha}}$ has conductor $p^{\alpha}$ and order dividing that of $H$.

The next result is critical in the determination of $F(X)$.

LEMMA 2. Let $H$ be a congruence group of conductor $m$ and order $f=2$, say $H=\{1, a\}$ modulo $m$ for some $a \in \mathbb{Z}_{m}^{*}$. Then $m=m_{0} m_{1}$ with $\left(m_{0}, m_{1}\right)=1$, where $H=H_{m_{0}} \cap H_{m_{1}}$, with $H_{m_{1}}=\{1\}$ (modulo $\left.m_{1}\right)$ and $H_{m_{0}}=\{ \pm 1\}$ (modulo $\left.m_{0}\right)$ or possibly $\left\{1, m_{0} / 2-1\right\}$ (modulo $\left.m_{0}\right)$ when $8 \mid m_{0}$. Moreover, the Gauss period $\zeta_{m}+\zeta_{m}^{a}$ is a conjugate of $\zeta_{m_{1}}\left(\zeta_{m_{0}}+\zeta_{m_{0}}^{a}\right)$.

Proof. Write $m$ as a product $p_{1}^{\alpha_{1}} \cdots p_{r}^{\alpha_{r}}$ of distinct prime powers, where $p_{1}<\cdots<p_{r}$ and $\alpha_{i}>0(1 \leq i \leq r)$. Since $H$ has conductor $m$ and order $f=2$, each congruence group $H_{p_{i}^{\alpha_{i}}}$ has conductor $p_{i}^{\alpha_{i}}(1 \leq i \leq r)$ with order equaling 1 or 2 . Let $m_{0}$ be the product of the prime powers $p_{i}^{\alpha_{i}}$ for which $H_{p_{i}}^{\alpha_{i}}$ has order 2 , and put $m_{1}=m / m_{0}$. (Note that if $p_{1}=2$ divides $m_{0}$ then necessarily $\alpha_{1}>2$.) Then $a \equiv 1\left(\bmod m_{1}\right)$ and $\operatorname{ord}_{p_{i}} \alpha_{i} a=2$ for each $p_{i} \mid m_{0}$. In particular, $a \equiv-1\left(\bmod p_{i}^{\alpha_{i}}\right)$ for any odd $p_{i} \mid m_{0}$ and $a \equiv-1$ or $2^{\alpha_{1}-1}-1$ $\left(\bmod 2^{\alpha_{1}}\right)$ should $p_{1}=2$ divide $m_{0}$. (The choice $a \equiv 2^{\alpha_{1}-1}+1\left(\bmod 2^{\alpha_{1}}\right)$ would contradict the fact that $H_{2^{\alpha_{1}}}$ has conductor $2^{\alpha_{1}}>4$.) It follows from the Chinese Remainder Theorem (as in (40) of [6]) that $a \equiv-1\left(\bmod m_{0}\right)$ or $a \equiv m_{0} / 2-1\left(\bmod m_{0}\right)$ respectively, so $H=H_{m_{0}} \cap H_{m_{1}}$, where $H_{m_{1}}=\{1\}$ modulo $m_{1}$ and $H_{m_{0}}=\{ \pm 1\}$ or $\left\{1, m_{0} / 2-1\right\}$ modulo $m_{0}$ according as $H_{2^{\alpha_{1}}}=\{ \pm 1\}$ or $\left\{1,2^{\alpha_{1}-1}-1\right\}$. The last statement of the proposition now follows readily from the Chinese Remainder Theorem, using the fact that $\zeta_{m_{1}}^{v}\left(\zeta_{m_{0}}^{w}+\zeta_{m_{0}}^{a w}\right)$, for $v \in \mathbb{Z}_{m_{1}}^{*}$ and $w \in \mathbb{Z}_{m_{0}}^{*} / H_{m_{0}}$, comprise a complete set of conjugates of $\zeta_{m_{1}}\left(\zeta_{m_{0}}+\zeta_{m_{0}}^{a}\right)$. 
Using the decomposition for $H$ in the lemma above, one can now express the reciprocal polynomial $F(X)$ in terms of the polynomials $F_{m_{0}}(X)$ or $G_{m_{0}}(X)$ appearing in Theorems 1 and 2 .

Proposition 3. Let $H$ be a congruence group of conductor $m$ and order $f=2$, say with $H=H_{m_{0}} \cap H_{m_{1}}$ as in Lemma 2 , where $m=m_{0} m_{1}$, $\left(m_{0}, m_{1}\right)=1$ and $H_{m_{0}}=\{ \pm 1\}$ modulo $m_{0}$ (or possibly $\left\{1, m_{0} / 2-1\right\}$ when $\left.8 \mid m_{0}\right)$. Then

$$
F(X)=\prod_{v \in \mathbb{Z}_{m_{1}}^{*}} F_{m_{0}}\left(\zeta_{m_{1}}^{v} X\right) \quad \text { or } \quad \prod_{v \in \mathbb{Z}_{m_{1}}^{*}} G_{m_{0}}\left(\zeta_{m_{1}}^{v} X\right)
$$

according as $H_{m_{0}}=\{ \pm 1\}$ or $\left\{1, m_{0} / 2-1\right\}$ modulo $m_{0}$. The corresponding sums $S_{n}$ of $n$th powers satisfy

$$
S_{n}=\mu\left(\frac{m_{1}}{\left(n, m_{1}\right)}\right) \frac{\phi\left(m_{1}\right)}{\phi\left(\frac{m_{1}}{\left(n, m_{1}\right)}\right)} S_{n}^{*} \quad(n>0),
$$

where $S_{n}^{*}$ are the $n$th power sums associated with $F_{m_{0}}(X)$ in Theorem 1 (Corollary 1 if $m_{0}=2^{\alpha_{1}}$ ) or $G_{m_{0}}(X)$ in Theorem 2, respectively.

Proof. Much of the proposition's assertions follow readily from Lemma 2 and its proof. To justify the formula for the power sums $S_{n}$ note that from Lemma 2,

$$
S_{n}=\sum_{v \in \mathbb{Z}_{m_{1}}^{*}, w \in \mathbb{Z}_{m_{0}}^{*} / H_{m_{0}}} \zeta_{m_{1}}^{n v}\left(\zeta_{m_{0}}^{w}+\zeta_{m_{0}}^{w a}\right)^{n}=\sum_{v \in \mathbb{Z}_{m_{1}}^{*}} \zeta_{m_{1}}^{n v} S_{n}^{*} \quad(n>0),
$$

where $S_{n}^{*}=\sum_{w \in \mathbb{Z}_{m_{0}}^{*} / H_{m_{0}}}\left(\zeta_{m_{0}}^{w}+\zeta_{m_{0}}^{w a}\right)^{n}(n>0)$ are the power sums associated with $F_{m_{0}}(X)$ or $G_{m_{0}}(X)$ according as $a \equiv-1$ or $m_{0} / 2-1\left(\bmod m_{0}\right)$. From (22),

$$
\sum_{v \in \mathbb{Z}_{m_{1}}^{*}} \zeta_{m_{1}}^{n v}=\mu(d) \frac{\phi\left(m_{1}\right)}{\phi(d)}
$$

where $\left(n, m_{1}\right)=m_{1} / d$, so the formula for $S_{n}$ given in the proposition follows.

I conclude this section with two examples illustrating Proposition 3.

Example 4. Consider $\theta_{1}=\zeta_{35}+\zeta_{35}^{29}$ in (1) where $H=\{1,29\} \bmod -$ ulo 35. Here $m_{0}=5$ and $m_{1}=7$ with $H_{m_{0}}=\{ \pm 1\}$ modulo 5 and $F_{5}(X)=1+X-X^{2}$ from Theorem 1 or direct calculation. One finds $\theta_{1}=\zeta_{7}^{3}\left(\zeta_{5}^{2}+\zeta_{5}^{-2}\right)$ with minimal polynomial

$$
\begin{aligned}
F(X)=\prod_{v \in \mathbb{Z}_{7}^{*}} F\left(\zeta_{7}^{v} X\right)= & 1-X+2 X^{2}-3 X^{3}+5 X^{4}-8 X^{5}+13 X^{6} \\
& +8 X^{7}+5 X^{8}+3 X^{9}+2 X^{10}+X^{11}+X^{12}
\end{aligned}
$$

from Proposition 3. Direct calculation of the power sums $S_{n}$ yields $S_{1}=1$, $S_{2}=-3, S_{3}=4, S_{4}=-7, S_{5}=11, S_{6}=-18, S_{7}=-174, S_{8}=-47$, 
$S_{9}=76, S_{10}=-123, S_{11}=199$ and $S_{12}=-322$ in agreement with the formula in Proposition 3.

ExAmPLE 5. Next consider $\theta_{1}=\zeta_{120}+\zeta_{120}^{19}$ in $(1)$ where $H=\{1,19\}$ modulo 120 . Here $m_{0}=40$ and $m_{1}=3$ from Lemma 2 with $H_{m_{0}}=\{1,19\}$ modulo 40. From Example $3, G_{40}(X)=1+8 X^{2}+19 X^{4}+12 X^{6}+X^{8}$. One finds $\theta_{1}=\zeta_{3}\left(\zeta_{40}^{27}+\zeta_{40}^{19 \cdot 27}\right)$ with minimal polynomial

$$
\begin{aligned}
F(X)=G_{40}\left(\zeta_{3} X\right) G_{40}\left(\zeta_{3}^{2} X\right)= & 1-8 X^{2}+45 X^{4}-128 X^{6} \\
& +264 X^{8}-212 X^{10}+125 X^{12}-12 X^{14}+X^{16}
\end{aligned}
$$

from Proposition 3.

3. Minimal polynomial for quadratic twists of $\zeta_{m}+\zeta_{m}^{-1}$. Here I consider certain twisted Gauss periods for odd $m$ of the form $\theta=i^{*} \sqrt{l}\left(\zeta_{m}+\right.$ $\left.(-1)^{(l-1) / 2} \zeta_{m}^{-1}\right)$, where $l \mid m^{\prime}$ with again $m^{\prime}=\prod_{p \mid m} p$ as in the previous section and $i^{*}=i^{(l-1)^{2} / 4}$. It is easy to see that $\theta$ generates $K=\mathbb{Q}\left(\zeta_{m}+\zeta_{m}^{-1}\right)$ since $\theta=\operatorname{Tr}_{\mathbb{Q}\left(\zeta_{m}\right) / K}\left(i^{*} \sqrt{l} \zeta_{m}\right)$ and

$$
\theta^{2}=(-1)^{(l-1) / 2} l\left(\zeta_{m}^{2}+\zeta_{m}^{-2}+(-1)^{(l-1) / 2} 2\right)
$$

already generates $K$. Now I wish to give formulas analogous to those in Theorem 1 for such quadratic twists, which ultimately depend on the Aurifeuille and Schinzel factors $[3,9,13]$ of the cyclotomic polynomial $\psi_{m^{\prime}}$ of the form

$$
\begin{aligned}
\psi_{m^{\prime}}\left((-1)^{(l-1) / 2} z^{2}\right)= & a_{0}+a_{2} z^{2}+\cdots+a_{\phi\left(m^{\prime}\right)} z^{\phi\left(m^{\prime}\right)} \\
& +\sqrt{l}\left(a_{1} z+a_{3} z^{3}+\cdots+a_{\phi\left(m^{\prime}\right)-1} z^{\phi\left(m^{\prime}\right)-1}\right) .
\end{aligned}
$$

The conjugates of $\theta$ are

$$
\theta_{v}=\left(\frac{v}{l}\right) i^{*} \sqrt{l}\left(\zeta_{m}^{v}+(-1)^{(l-1) / 2} \zeta_{m}^{-v}\right) \quad\left(v \in \mathbb{Z}_{m}^{*} /( \pm 1)\right)
$$

with power sums $S_{n}$ equaling

$$
\begin{aligned}
l^{(n-1) / 2} m_{+} \sum_{t=1,(t, l)=1, t \text { odd }}^{\left[n m^{\prime} / m\right]}(-1)^{(l-1)\left(1+m_{+} t / m^{\prime}\right) / 4} & \\
& \times \delta_{m, l}(t)\left(\begin{array}{c}
n \\
\left(n-m t / m^{\prime}\right) / 2
\end{array}\right),
\end{aligned}
$$

if $n$ is odd, where

$$
\delta_{m, l}(t)=\left(\frac{l t / m^{\prime}}{l}\right) \mu\left(\frac{m_{-}}{\left(m t / m^{\prime}, m_{-}\right)}\right) \frac{\phi\left(m_{-}\right)}{\phi\left(\frac{m_{-}}{\left(m t / m^{\prime}, m_{-}\right)}\right)},
$$

or

$$
l^{n / 2}\left(\frac{\phi(m)}{2}\left(\begin{array}{c}
n \\
n / 2
\end{array}\right)+\sum_{d \mid m} \mu(d) \frac{\phi(m)}{\phi(d)} \sum_{t=1,(t, d)=1}^{[d n / 2 m]}(-1)^{(l-1) t / 2}\left(\begin{array}{c}
n \\
n / 2-m t / d
\end{array}\right)\right)
$$


if $n$ is even. Here $m$ is uniquely expressed in the form $m=m_{+} m_{-}$where $\left(m_{-}, l\right)=1$ and $m_{+}>0$ is divisible only by primes dividing $l$. The above formulas for $S_{n}$ are readily obtained directly as in (23) using the expansions

$$
\theta_{1}^{n}=l^{n / 2}\left(\begin{array}{c}
n \\
n / 2
\end{array}\right)+l^{n / 2} \sum_{j=0}^{n / 2-1}(-1)^{(l-1)(n+2 j) / 4}\left(\begin{array}{c}
n \\
j
\end{array}\right)\left(\zeta_{m}^{n-2 j}+\zeta_{m}^{2 j-n}\right)
$$

if $n$ is even, or

$$
\begin{aligned}
(-1)^{(l-1)(n-1) / 4} l^{(n-1) / 2} i^{*} \sqrt{l} \sum_{j=0}^{(n-1) / 2}\left(\begin{array}{l}
n \\
j
\end{array}\right) & (-1)^{(l-1) j / 2} \\
& \times\left(\zeta_{m}^{n-2 j}+(-1)^{(l-1) / 2} \zeta_{m}^{2 j-n}\right)
\end{aligned}
$$

if $n$ is odd, from the binomial theorem together with equation (22), the fact that

$$
\sum_{v \in \mathbb{Z}_{m}^{*} /( \pm 1)}\left(\left(\frac{v}{l}\right) \zeta_{m}^{t v}+(-1)^{(l-1) / 2} \zeta_{m}^{-t v}\right)=\sum_{v \in \mathbb{Z}_{m}^{*}}\left(\frac{v}{l}\right) \zeta_{m}^{t v},
$$

and the lemma below. Details of the calculation, similar to that in establishing (23), are left to the reader.

LeMma 3. With notation as above,

$$
\sum_{x \in \mathbb{Z}_{m}^{*}}\left(\frac{x}{l}\right) \zeta_{m}^{t x}=i^{*} \sqrt{l} \frac{m_{+}}{l}\left(\frac{l t / m}{l}\right) \mu\left(\frac{m_{-}}{\left(t, m_{-}\right)}\right) \frac{\phi\left(m_{-}\right)}{\phi\left(\frac{m_{-}}{\left(t, m_{-}\right)}\right)}
$$

if $\left(m_{+}, t\right)=m_{+} / l$ and 0 otherwise.

Proof. First note that

$$
\sum_{x \in \mathbb{Z}_{m}^{*}} \zeta_{m}^{t x}=\mu(d) \frac{\phi(m)}{\phi(d)}
$$

where $(t, m)=m / d$ from (22). Applying the result of problem 4, p. 336, in [2] with $m=m_{+} m_{-}$, one finds

$$
\sum_{x \in \mathbb{Z}_{m}^{*}}\left(\frac{x}{l}\right) \zeta_{m}^{t x}=\left(\frac{m_{-}}{l}\right) \sum_{x \in \mathbb{Z}_{m_{+}}^{*}}\left(\frac{x}{l}\right) \zeta_{m_{+}}^{t x} \cdot \sum_{x \in \mathbb{Z}_{m_{-}}^{*}} \zeta_{m_{-}}^{t x} .
$$

The first sum in the product on the right is non-vanishing only when $\left(t, m_{+}\right)$ $=m_{+} / l$, since otherwise in the factorization of $\sum_{x \in \mathbb{Z}_{m_{+}}^{*}}\left(\frac{x}{l}\right) \zeta_{m_{+}}^{t x}$ as a product of Gauss sums defined modulo the distinct prime powers dividing $m_{+}$, at least one such component will be zero (by problem 4, p. 336 in [2] again, and the fact that any imprimitive Gauss sum defined modulo a prime power vanishes). If $\left(t, m_{+}\right)=m_{+} / l$, say $t=m_{+} v / l$ with $(v, l)=1$, then $\sum_{x \in \mathbb{Z}_{m_{+}}^{*}}\left(\frac{x}{l}\right) \zeta_{m_{+}}^{t x}$ is just $m_{+} / l$ copies of $\sum_{x \in \mathbb{Z}_{l}^{*}}\left(\frac{x}{l}\right) \zeta_{l}^{v x}$, so equals $i^{*} \sqrt{l} \frac{m_{+}}{l}\left(\frac{l t / m_{+}}{l}\right)$. The second 
sum in the product equals

$$
\mu\left(\frac{m_{-}}{\left(t, m_{-}\right)}\right) \frac{\phi\left(m_{-}\right)}{\phi\left(\frac{m_{-}}{\left(t, m_{-}\right)}\right)}
$$

by my initial observation. The result of the lemma now follows since

$$
\left(\frac{m_{-}}{l}\right)\left(\frac{l t / m_{+}}{l}\right)=\left(\frac{l t / m}{l}\right) .
$$

My aim here is to find a formula for the minimal polynomial of $\theta$, or more precisely for the reciprocal polynomial $P_{m, l}(X)$, analogous to that for $F_{m}(X)$ in Section 2, whose zeros are the reciprocals of $\theta_{v}$ in (25). To this end I first find an expression for the polynomial $P(X)$ with zeros $\left\{ \pm \theta_{v}^{-1} \mid v \in\right.$ $\left.\mathbb{Z}_{m}^{*} /( \pm 1)\right\}$. From $(24)$, one has $\left(\zeta_{m}^{2}+\zeta_{m}^{-2}\right)^{-1}=(-1)^{(l-1) / 2} l \theta^{-2} /\left(1-2 l \theta^{-2}\right)$, a zero of $F_{m}(X)$, so

$$
\begin{aligned}
P(X)= & \left(1-2 l X^{2}\right)^{\phi(m) / 2} F_{m}\left((-1)^{(l-1) / 2} l X^{2} /\left(1-2 l X^{2}\right)\right) \\
= & \left(\frac{1-2 l X^{2}+\sqrt{1-4 l X^{2}}}{2}\right)^{\phi(m) / 2} \\
& \times \psi_{m^{\prime}}\left(\left(\frac{1-2 l X^{2}-\sqrt{1-4 l X^{2}}}{(-1)^{(l-1) / 2} l X^{2}}\right)^{m / m^{\prime}}\right) \\
= & \left(\frac{1+\sqrt{1-4 l X^{2}}}{2}\right)^{\phi(m)} \psi_{m^{\prime}}\left((-1)^{(l-1) / 2}\left(\frac{1-\sqrt{1-4 l X^{2}}}{2 \sqrt{l})^{2 m / m^{\prime}}}\right)\right.
\end{aligned}
$$

from $(20)$ since $\left(\left(1 \pm \sqrt{1-4 l X^{2}}\right) / 2\right)^{2}=\left(1-2 l X^{2} \pm \sqrt{1-4 l X^{2}}\right) / 2$. For convenience I write

$$
P(X)=E_{l}(X)^{\phi(m)} \psi_{m^{\prime}}\left((-1)^{(l-1) / 2} A_{l}(X)^{2 m / m^{\prime}}\right)
$$

where $E_{l}(X)=\left(1+\sqrt{1-4 l X^{2}}\right) / 2, \bar{E}_{l}(X)=\left(1-\sqrt{1-4 l X^{2}}\right) / 2$ and $A_{l}(X)=$ $\left(1-\sqrt{1-4 l X^{2}}\right) /(2 \sqrt{l} X)$. Now $P(X)=P_{m, l}(X) \cdot P_{m, l}(-X)$ over $\mathbb{Z}[X]$, so the strategy is to find the correct factor of $P(X)$ in $(28)$.

Suppose $\psi_{m^{\prime}}\left((-1)^{(l-1) / 2} z^{2}\right)$ factors in $\mathbb{Q}(\sqrt{l})$ as $g_{m^{\prime}, l}(z) g_{m^{\prime}, l}(-z)$ with $g_{m^{\prime}, l}(z)$ self-reciprocal and of the form

$$
\begin{aligned}
g_{m^{\prime}, l}(z)= & a_{0}+a_{2} z^{2}+\cdots+a_{\phi\left(m^{\prime}\right)} z^{\phi\left(m^{\prime}\right)} \\
& +\sqrt{l}\left(a_{1} z+a_{3} z^{3}+\cdots+a_{\phi\left(m^{\prime}\right)-1} z^{\phi\left(m^{\prime}\right)-1}\right)
\end{aligned}
$$

for integers $a_{j}\left(0 \leq j \leq \phi\left(m^{\prime}\right)\right)$. Then $a_{\phi\left(m^{\prime}\right)-j}=a_{j}\left(0 \leq j \leq \phi\left(m^{\prime}\right) / 2\right)$ and $E_{l}(X)^{\phi(m) / 2} \cdot g_{m^{\prime}, l}\left(A_{l}(X)^{m / m^{\prime}}\right)$ is a polynomial in $\mathbb{Z}[X]$. In fact, since $E_{l}(X) A_{l}(X)=\sqrt{l} X$ and $\sqrt{l} X A_{l}(X)=\bar{E}_{l}(X)$, this polynomial is 
$E_{l}(X)^{\frac{m}{m^{\prime}} \frac{\phi\left(m^{\prime}\right)}{2}}$

$$
\times\left(\sum_{j=0}^{\phi\left(m^{\prime}\right) / 2} a_{2 j}\left(A_{l}(X)^{m / m^{\prime}}\right)^{2 j}+\sum_{j=1}^{\phi\left(m^{\prime}\right) / 2} a_{2 j-1} \sqrt{l}\left(A_{l}(X)^{m / m^{\prime}}\right)^{2 j-1}\right),
$$

which equals

$$
\begin{aligned}
& a_{\phi\left(m^{\prime}\right) / 2} l^{[(\phi(m)+2) / 4]} X^{\phi(m) / 2} \\
& {\left[\left(\phi\left(m^{\prime}\right)-2\right) / 4\right]} \\
& +\sum_{j=0} a_{2 j}\left(l X^{2}\right)^{m j / m^{\prime}} C_{\frac{m}{m^{\prime}}\left(\phi\left(m^{\prime}\right) / 2-2 j\right)}(\sqrt{l} X) \\
& +\sum_{j=1}^{\left[\phi\left(m^{\prime}\right) / 4\right]} a_{2 j-1} l^{\left(m(2 j-1) / m^{\prime}+1\right) / 2} X^{m(2 j-1) / m^{\prime}} C_{\frac{m}{m^{\prime}}\left(\phi\left(m^{\prime}\right) / 2-2 j+1\right)}(\sqrt{l} X) .
\end{aligned}
$$

To find such a factor $g_{m^{\prime}, l}(z)$ first consider

$$
g(x)=\prod_{v \in \mathbb{Z}_{m^{\prime}}^{*}}\left(1-\left(\frac{v}{l}\right) \zeta_{m^{\prime}}^{v} X\right)
$$

a polynomial over $\mathbb{Q}\left(i^{*} \sqrt{l}\right)$ with power sums given by (see Lemma 3 )

$$
\begin{aligned}
\sum_{v \in \mathbb{Z}_{m^{\prime}}^{*}} & \left(\frac{v}{l}\right) \zeta_{m^{\prime}}^{v n} \\
& = \begin{cases}i^{*} \sqrt{l}\left(\frac{\ln / m^{\prime}}{l}\right) \mu\left(\frac{m^{\prime} / l}{\left(n, m^{\prime} / l\right)}\right) \phi\left(\left(n, m^{\prime} / l\right)\right) & \text { if }(n, l)=1, \\
0 & \text { if }(n, l) \neq 1\end{cases}
\end{aligned}
$$

when $n$ is odd, or by

$$
\sum_{v \in \mathbb{Z}_{m^{\prime}}^{*}} \zeta_{m^{\prime}}^{v n}=\mu(d) \phi\left(m^{\prime} / d\right)
$$

when $n$ is even, where $\left(n, m^{\prime}\right)=m^{\prime} / d$. I assert that $g_{m^{\prime}, l}(z)=g(\varepsilon z)$, where

$$
\varepsilon=(-1)^{(l-1)\left(1-m / m^{\prime}\right) / 4} i^{*}= \begin{cases}1 & \text { if } l \equiv 1(\bmod 4), \\ (-1)^{\left(1-m / m^{\prime}\right) / 2} i & \text { if } l \equiv 3(\bmod 4),\end{cases}
$$

has the desirable characteristics in (29). From (31) its associated power sums for odd $n$ are

$$
S_{n}= \begin{cases}i^{*} \varepsilon^{n}\left(\frac{\ln / m^{\prime}}{l}\right) \mu\left(\frac{m^{\prime} / l}{\left(n, m^{\prime} / l\right)}\right) \phi\left(\left(n, m^{\prime} / l\right)\right) \sqrt{l} & \text { if }(n, l)=1 \\ 0 & \text { otherwise }\end{cases}
$$

and

$$
S_{n}=(-1)^{(l-1) n / 4} \mu(d) \phi\left(m^{\prime} / d\right) \quad \text { if } n \text { is even, }
$$


where $\left(n, m^{\prime}\right)=m^{\prime} / d$. From Newton's identities (6) one readily finds that $g_{m^{\prime}, l}(z)$ has the form (29) with $a_{j}$ satisfying a polynomial dependence on $l$ of degree $\leq[j / 2]$. Furthermore, $a_{\phi\left(m^{\prime}\right)-j}=a_{j}$ since $g_{m^{\prime}, l}(z)$ is seen to be self-reciprocal. In fact, $g_{m^{\prime}, m^{\prime}}(z)$ is just the polynomial $L_{m^{\prime}}(z)$ or $L_{m^{\prime}}(-z)$ in equation (24) in [3], so is expressible in terms of the Aurifeuille factors of $\psi_{m^{\prime}}\left((-1)^{\left(m^{\prime}-1\right) / 2} z\right)$. More generally, $g_{m^{\prime}, l}(z)$ is seen to be expressible in terms of the Schinzel factors [13] of $\psi_{m^{\prime}}\left((-1)^{(l-1) / 2} z\right)$. Brent [3] gives an efficient algorithm to compute $g_{m^{\prime}, m^{\prime}}(z)$ from Newton's identities, basically due to Dirichlet, that readily generalizes to compute $g_{m^{\prime}, l}(z)$ here.

I assert that $P_{m, l}(X)=E_{l}(X)^{\phi(m) / 2} g_{m^{\prime}, l}\left(A_{l}(X)^{m / m^{\prime}}\right)$ is the correct choice with zeros $\theta_{v}^{-1}$ for $\theta_{v}$ in (25). Indeed:

TheOREM 3. Let $g_{m^{\prime}, l}(z)$ be the self-reciprocal polynomial of the form (29) and of degree $\phi\left(m^{\prime}\right)$ over $\mathbb{Q}(\sqrt{l})$ determined from the power sums in (33). Then $P_{m, l}(X)$ is given by (30) with coefficients $c_{r}$ for $X^{r}$ satisfying

$$
\begin{aligned}
c_{r}= & l^{[(r+1) / 2]} \sum_{j=0, j \equiv r(\bmod 2)}^{\left[m^{\prime} r / m\right]}(-1)^{t_{j}} a_{j} \\
& \times \frac{\frac{m}{m^{\prime}}\left(\frac{\phi\left(m^{\prime}\right)}{2}-j\right)}{\frac{m}{m^{\prime}}\left(\frac{\phi\left(m^{\prime}\right)}{2}-j\right)-t_{j}}\left(\begin{array}{c}
\frac{m}{m^{\prime}}\left(\frac{\phi\left(m^{\prime}\right)}{2}-j\right)-t_{j} \\
t_{j}
\end{array}\right)
\end{aligned}
$$

for $1 \leq r<\phi(m) / 2$, and

$$
\begin{aligned}
c_{\phi(m) / 2}= & l^{[(\phi(m)+2) / 4]} \\
& \times\left(a_{\phi\left(m^{\prime}\right) / 2}+(-1)^{\left[\left(\phi\left(m^{\prime}\right)+2\right) / 4\right]} \sum_{j=0, j \equiv \phi(m) / 2(\bmod 2)}^{\phi\left(m^{\prime}\right) / 2-2}(-1)^{[(j+1) / 2]} 2 a_{j}\right),
\end{aligned}
$$

where $t_{j}=\left(r-m j / m^{\prime}\right) / 2$.

Proof. In view of the remarks already made it suffices to show that $E_{l}(X)^{\phi(m) / 2} g_{m^{\prime}, l}\left(A_{l}(X)^{m / m^{\prime}}\right)$, which yields the polynomial expression in (30) above, has associated power sums matching those in (26). Again, expanding $\log (1-T)$ about $T=0$, one finds $\log E_{l}(X)^{\phi(m) / 2} g_{m^{\prime}, l}\left(A_{l}(X)^{m / m^{\prime}}\right)$ equals

$$
\begin{aligned}
\frac{\phi(m)}{2} \log E_{l}(X)+\sum_{w \in \mathbb{Z}_{m^{\prime}}^{*}} \log \left(1-\left(\frac{w}{l}\right) \varepsilon \zeta_{m^{\prime}}^{w} A_{l}(X)^{m / m^{\prime}}\right) \\
=-\frac{\phi(m)}{2} \sum_{n=1}^{\infty}\left(\begin{array}{c}
2 n \\
n
\end{array}\right) \frac{l^{n} X^{2 n}}{2 n}-\sum_{w \in \mathbb{Z}_{m^{\prime}}^{*}} \sum_{v=1}^{\infty} \frac{\varepsilon^{v}}{v}\left(\frac{w}{l}\right) \zeta_{m^{\prime}}^{w v} A_{l}(X)^{m v / m^{\prime}}
\end{aligned}
$$




$$
=-\frac{\phi(m)}{2} \sum_{n=1}^{\infty}\left(\begin{array}{c}
2 n \\
n
\end{array}\right) \frac{l^{n} X^{2 n}}{2 n}-\sum_{v=1}^{\infty} \frac{\varepsilon^{v}}{v} A_{l}(X)^{m v / m^{\prime}} \sum_{w \in \mathbb{Z}_{m^{\prime}}^{*}}\left(\frac{w}{l}\right)^{v} \zeta_{m^{\prime}}^{w v} .
$$

In view of (31) and Lemma 1, this last expression is seen to have the coefficient of $X^{n}$ equal to

$$
\begin{aligned}
-\frac{\phi(m)}{2 n}\left(\begin{array}{c}
n \\
n / 2
\end{array}\right) l^{n / 2} & -\frac{1}{n} \sum_{d \mid m^{\prime}} \mu(d) \\
& \times \frac{\phi\left(m^{\prime}\right)}{\phi(d)} \sum_{t=1,(t, d)=1}^{[d n / 2 m]}(-1)^{(l-1) t / 2} l^{n / 2} \frac{m}{m^{\prime}}\left(\begin{array}{c}
n \\
n / 2-m t / d
\end{array}\right)
\end{aligned}
$$

if $n$ is even, or

$$
\begin{array}{r}
-\frac{l^{(n-1) / 2}}{n} \sum_{t=1,(t, l)=1, t \text { odd }}^{\left[m^{\prime} n / m\right]} i^{*} \varepsilon^{t}\left(\frac{l t / m^{\prime}}{l}\right) \mu\left(\frac{m^{\prime} / l}{\left(t, m^{\prime} / l\right)}\right) \frac{\phi\left(m^{\prime} / l\right)}{\phi\left(\frac{m^{\prime} / l}{\left(t, m^{\prime} / l\right)}\right)} \\
\times \frac{l m}{m^{\prime}}\left(\begin{array}{c}
n \\
n / 2-m t / 2 m^{\prime}
\end{array}\right)
\end{array}
$$

if $n$ is odd. Since for $(t, l)=1$,

$$
\left(m t / m^{\prime}, m_{-}\right)=\left(\frac{m_{-} t}{m^{\prime} / l}, m_{-}\right)=\frac{m_{-}}{m^{\prime} / l}\left(t, m^{\prime} / l\right)
$$

one finds

$$
\frac{m^{\prime} / l}{\left(t, m^{\prime} / l\right)}=\frac{m_{-}}{\left(m t / m^{\prime}, m_{-}\right)} \quad \text { and } \quad \frac{\phi\left(m^{\prime} / l\right)}{\phi\left(\frac{m^{\prime} / l}{\left(t, m^{\prime} / l\right)}\right)}=\frac{m^{\prime} / l}{m_{-}} \frac{\phi\left(m_{-}\right)}{\phi\left(\frac{m_{-}}{\left(m t / m^{\prime}, m_{-}\right)}\right)}
$$

so this last expression for odd $n$ equals

$$
-\frac{l^{(n-1) / 2} m_{+}}{n} \sum_{t=1,(t, l)=1, t \text { odd }}^{\left[n m^{\prime} / m\right]} i^{*} \varepsilon^{t} \delta_{m, l}(t)\left(\begin{array}{c}
n \\
\left(n-m t / m^{\prime}\right) / 2
\end{array}\right)
$$

with $\delta_{m, l}(t)$ as in (26). But for $t$ odd, $i^{*} \varepsilon^{t}=(-1)^{(l-1)\left(1+m t / m^{\prime}\right) / 4}$ from $(32)$, so the polynomial $E_{l}(X)^{\phi(m) / 2} g_{m^{\prime}, l}\left(A_{l}(X)^{m / m^{\prime}}\right)$ has associated power sums as in (26).

The formulas for the coefficients $c_{r}$ are obtained in a straightforward fashion from the expression (30). This completes the proof of the theorem.

Next I give a few examples to illustrate Theorem 3.

Example 6. Consider $\theta_{1}=i \sqrt{15}\left(\zeta_{15}-\zeta_{15}^{-1}\right)$ in $(25)$. Here $l=m_{+}=$ $m=m^{\prime}=15$ and $m_{-}=1$ with $\psi_{15}(x)=1-x+x^{3}-x^{4}+x^{5}-x^{7}+x^{8}$. One finds

$$
g_{15,15}(z)=1+8 z^{2}+13 z^{4}+8 z^{6}+z^{8}+\sqrt{15}\left(z+3 z^{3}+3 z^{5}+z^{7}\right)
$$


is the correct "Aurifeuille" factor of $\psi_{15}\left(-z^{2}\right)$ satisfying (32). Indeed direct computation of $P_{15,15}(X)$ yields

$$
P_{15,15}(X)=1+15 X+60 X^{2}-225 X^{4}
$$

whose coefficients agree with those obtained from (34). Note that $\theta=$ $i \sqrt{15}\left(\zeta_{45}-\zeta_{45}^{-1}\right)$ with $l=m^{\prime}=15, m_{+}=m=45$ and $m_{-}=1$ requires the conjugate factor

$$
g_{15,15}(z)=1+8 z^{2}+13 z^{4}+8 z^{6}+z^{8}-\sqrt{15}\left(z+3 z^{3}+3 z^{5}+z^{7}\right)
$$

in the computation of

$$
\begin{aligned}
P_{45,15}(X)= & 1-180 X^{2}-225 X^{3}+54 \cdot 15^{2} x^{4}+9 \cdot 15^{3} X^{5}-104 \cdot 15^{3} X^{6} \\
& -27 \cdot 15^{4} X^{7}+57 \cdot 15^{4} X^{8}+27 \cdot 15^{5} X^{9}+36 \cdot 15^{5} X^{10}-15^{6} X^{12} .
\end{aligned}
$$

EXAMPLE 7. Next consider $\theta_{1}=i \sqrt{3}\left(\zeta_{45}-\zeta_{45}^{-1}\right)$ in (25) with $m=45$, $l=3, m_{+}=9, m^{\prime}=15$ and $m_{-}=5$, and again $\psi_{15}(x)=1-x+x^{3}-x^{4}+$ $x^{5}-x^{7}+x^{8}$. One finds here that

$$
g_{15,3}(z)=1+2 z^{2}+z^{4}+2 z^{6}+z^{8}-\sqrt{3}\left(z+z^{3}+z^{5}+z^{7}\right)
$$

is the correct "Schinzel" factor of $\psi_{15}\left(-z^{2}\right)$ satisfying (32). Direct computation of the power sums $S_{n}$ yields $S_{1}=0, S_{2}=72, S_{3}=27, S_{4}=3^{4} \cdot 8$, $S_{5}=3^{4} \cdot 5, S_{6}=3^{4} \cdot 79, S_{7}=3^{6} \cdot 7, S_{8}=3^{5} \cdot 272, S_{9}=3^{7} \cdot 28, S_{10}=3^{8} \cdot 107$, $S_{11}=3^{8} \cdot 110$ and $S_{12}=3^{8} \cdot 1159$, with

$$
\begin{aligned}
P_{45,3}(X)= & 1-36 X^{2}-9 X^{3}+3^{5} \cdot 2 X^{4}+3^{5} X^{5}-3^{3} \cdot 110 X^{6} \\
& -3^{7} X^{7}+3^{4} \cdot 93 X^{8}+3^{5} \cdot 29 X^{9}-3^{7} \cdot 2 X^{10}-3^{7} \cdot 2 X^{11}-3^{6} X^{12}
\end{aligned}
$$

in agreement with the formulas in Theorem 3. If instead one takes $\theta_{1}=$ $\sqrt{5}\left(\zeta_{45}+\zeta_{45}^{-1}\right)$ in $(25)$ so $m=45, l=m_{+}=5, m^{\prime}=15$ and $m_{-}=9$, then the correct "Schinzel" factor of $\psi_{15}\left(z^{2}\right)$ satisfying (32) is

$$
g_{15,5}(z)=1+2 z^{2}+3 z^{4}+2 z^{6}+z^{8}-\sqrt{5}\left(z+z^{3}+z^{5}+z^{7}\right) .
$$

Direct computation yields

$$
\begin{aligned}
P_{45,5}(X)= & 1-60 X^{2}-25 X^{3}+5^{2} \cdot 54 X^{4}+5^{3} \cdot 9 X^{5}-5^{4} \cdot 22 X^{6}-5^{4} \cdot 27 X^{7} \\
& +5^{4} \cdot 93 X^{8}+5^{5} \cdot 29 X^{9}-5^{5} \cdot 18 X^{10}-5^{6} \cdot 6 X^{11}+5^{6} X^{12}
\end{aligned}
$$

whose coefficients agree with those determined from (34).

EXAMPLE 8. Now consider $\theta_{1}=\sqrt{21}\left(\zeta_{21}+\zeta_{21}^{-1}\right)$ in $(25)$. Here $l=m_{+}=$ $m=m^{\prime}=21$ and $m_{-}=1$, with $\psi_{21}(x)=1-x+x^{3}-x^{4}+x^{6}-x^{8}+x^{9}-$ $x^{11}+x^{12}$. One finds here that

$$
\begin{aligned}
g_{21,21}(z)= & 1+10 z^{2}+13 z^{4}+7 z^{6}+13 z^{8}+10 z^{10}+x^{12} \\
& -\sqrt{21}\left(x+3 x^{3}+2 x^{5}+2 x^{7}+3 x^{9}+x^{11}\right)
\end{aligned}
$$


is the correct "Aurifeuille" factor of $\psi_{21}\left(z^{2}\right)$ satisfying (32). Direct computation yields

$$
P_{21,21}(X)=1-21 X+84 X^{2}+882 X^{3}-7938 X^{4}+18522 X^{5}-9261 X^{6},
$$

whose coefficients agree with those found from $(34)$.

If one considers instead $\theta_{1}=i \sqrt{7}\left(\zeta_{21}-\zeta_{21}^{-1}\right)$ in (25), so $l=m_{+}=7$, $m^{\prime}=m=21$ and $m_{-}=3$, one finds that

$$
\begin{aligned}
g_{21,7}(x)= & 1+4 z^{2}-z^{4}-7 z^{6}-z^{8}+4 z^{10}+z^{12} \\
& +\sqrt{7}\left(z+z^{3}-2 z^{5}-2 z^{7}+z^{9}+z^{11}\right)
\end{aligned}
$$

is the correct "Schinzel" factor of $\psi_{21}\left(-z^{2}\right)$ satisfying (32) with

$$
P_{21,7}(X)=1+7 X-14 X^{2}-7^{2} \cdot 4 X^{3}-7^{2} \cdot 8 X^{4}+7^{3} X^{6}
$$

from (34).

The special case $m=p^{\alpha}$ warrants special consideration. Here I simply write $P_{p^{\alpha}}(X)$ for $P_{p^{\alpha}, p}(X)$.

Corollary 3. For an odd prime $p, P_{p^{\alpha}}(X)$ has the form

$$
\begin{aligned}
a_{(p-1) / 2} p^{p^{\alpha-1}[(p+1) / 4]} X^{p^{\alpha-1}(p-1) / 2} & \\
& +\sum_{j=0}^{[(p-3) / 4]} a_{2 j}\left(p X^{2}\right)^{p^{\alpha-1} j} C_{p^{\alpha-1}\left(\frac{p-1}{2}-2 j\right)}(\sqrt{p} X) \\
& +\sum_{j=1}^{[(p-1) / 4]} a_{2 j-1} p^{\left(p^{\alpha-1}(2 j-1)+1\right) / 2} X^{p^{\alpha-1}(2 j-1)} C_{p^{\alpha-1}\left(\frac{p-1}{2}-2 j+1\right)}(\sqrt{p} X),
\end{aligned}
$$

with coefficient $c_{r}$ for $X^{r}$ satisfying

$$
\begin{aligned}
c_{r}= & p^{[(r+1) / 2]} \sum_{j=0, j \equiv r(\bmod 2)}^{\left[r p^{1-\alpha}\right]}(-1)^{t_{j}} a_{j} \\
& \times \frac{p^{\alpha-1}\left(\frac{p-1}{2}-j\right)}{p^{\alpha-1}\left(\frac{p-1}{2}-j\right)-t_{j}}\left(\begin{array}{c}
p^{\alpha-1}\left(\frac{p-1}{2}-j\right)-t_{j} \\
t_{j}
\end{array}\right)
\end{aligned}
$$

for $1 \leq r<\phi\left(p^{\alpha}\right) / 2$, where $t_{j}=\left(r-p^{\alpha-1} j\right) / 2$, and with

$$
c_{\phi\left(p^{\alpha}\right) / 2}= \begin{cases}\left(\frac{2}{p}\right) p^{\phi\left(p^{\alpha}\right) / 4} & \text { if } p \equiv 1(\bmod 4), \\ (-1)^{N}\left(\frac{2}{p}\right)(-p)^{\left(\phi\left(p^{\alpha}\right)+2\right) / 4} & \text { if } p \equiv 3(\bmod 4),\end{cases}
$$

where $N$ is the number of quadratic non-residues of $p$ in $(0, p / 2)$.

Proof. I need only justify the determination of the last coefficient $c_{\phi\left(p^{\alpha}\right) / 2}$ $=(-1)^{\phi\left(p^{\alpha}\right) / 2} N_{K / \mathbb{Q}}\left(i^{*} \sqrt{p}\left(\zeta_{p^{\alpha}}+(-1)^{(p-1) / 2} \zeta_{p^{\alpha}}^{-1}\right)\right)$, where $K=\mathbb{Q}\left(\zeta_{p^{\alpha}}+\zeta_{p^{\alpha}}^{-1}\right)$. 
For $p \equiv 1(\bmod 4)$, one immediately has $c_{\phi\left(p^{\alpha}\right) / 2}=\left(\frac{2}{p}\right) p^{\phi\left(p^{\alpha}\right) / 4}$ since $N_{K / \mathbb{Q}}\left(\zeta_{p^{\alpha}}+\zeta_{p^{\alpha}}^{-1}\right)=\left(\frac{2}{p}\right)$ from $(19)$. For $p \equiv 3(\bmod 4)$, one notes that (see problem 14, p. 355 in [2]; see also (35) below)

$$
\prod_{v=1, p \nmid v}^{\left[p^{\alpha} / 2\right]}\left(\zeta_{p^{\alpha}}^{v}-\zeta_{p^{\alpha}}^{-v}\right)=\prod_{v=1}^{[p / 2]}\left(\zeta_{p}-\zeta_{p}^{-v}\right)=i \sqrt{p}\left(\frac{-2}{p}\right),
$$

so that

$$
c_{\phi\left(p^{\alpha}\right) / 2}=-\prod_{v=1, p \nmid v}^{\left[p^{\alpha} / 2\right]} i \sqrt{p}\left(\frac{v}{p}\right)\left(\zeta_{p^{\alpha}}^{v}-\zeta_{p^{\alpha}}^{-v}\right)=\left(\frac{2}{p}\right)(-1)^{N}(-p)^{\left(\phi\left(p^{\alpha}\right)+2\right) / 4},
$$

where $N$ counts the number of times $\left(\frac{v}{p}\right)=-1$ for $1 \leq v \leq(p-1) / 2$.

Actually it is no more difficult to determine the last coefficient $c_{\phi(m) / 2}$ for the polynomial $P_{m, l}(X)$ in Theorem 3 in general. For odd composite $m^{\prime}, c_{\phi(m) / 2}$ is the norm of $\theta_{1}$ in (25) so equals $\pm l^{\phi(m) / 4}$ since $i^{*}\left(\zeta_{m}+\right.$ $\left.(-1)^{(l-1) / 2} \zeta_{m}^{-1}\right)$ is a unit of $K$. The correct sign is given by

Proposition 4. For odd composite $m^{\prime}$ in Theorem 3,

$$
c_{\phi(m) / 2}=(-1)^{N} l^{\phi(m) / 4}
$$

where $N$ counts the number of reduced residues $v$ modulo $m^{\prime}$ in $\left(0, m^{\prime} / 2\right)$ with $\left(\frac{v}{l}\right)=-1$.

Proof. First note that for any integer $a$ with $\left(a, m^{\prime}\right)=1$,

$$
\prod_{v \in \mathbb{Z}_{m}^{*}, v \equiv a\left(\bmod m^{\prime}\right)}\left(\zeta_{m}^{v} \pm \zeta_{m}^{-v}\right)=\prod_{\lambda=0}^{m / m^{\prime}-1}\left(\zeta_{m / m^{\prime}}^{\lambda} \zeta_{m}^{a} \pm \zeta_{m / m^{\prime}}^{-\lambda} \zeta_{m}^{-a}\right)=\zeta_{m^{\prime}}^{a} \pm \zeta_{m^{\prime}}^{-a}
$$

since $\zeta_{m}^{a+\lambda m^{\prime}}=\zeta_{m / m^{\prime}}^{\lambda} \cdot \zeta_{m}^{a}$ for $0 \leq \lambda<m / m^{\prime}$. Moreover, I assert here that

$$
\prod_{v=1,\left(v, m^{\prime}\right)=1}^{\left(m^{\prime}-1\right) / 2} 2 \sin \frac{2 \pi v}{m^{\prime}}=\prod_{v=1,\left(v, m^{\prime}\right)=1}^{\left(m^{\prime}-1\right) / 2} 2 \cos \frac{2 \pi v}{m^{\prime}}=1
$$

since $m^{\prime}$ is odd and composite. To verify (36) observe that up to sign $\prod 2 \sin \left(2 \pi v / m^{\prime}\right)$ is the norm from $K$ to $\mathbb{Q}$ of the unit $i\left(\zeta_{m^{\prime}}-\zeta_{m^{\prime}}^{-1}\right)$, so it equals \pm 1 . But $2 \sin \left(2 \pi v / m^{\prime}\right)>0$ for $1 \leq v \leq\left(m^{\prime}-1\right) / 2$, so that the product must be 1 and hence also the product of its conjugates $2 \sin \left(4 \pi v / m^{\prime}\right)$ for $1 \leq v \leq\left(m^{\prime}-1\right) / 2$. Since

$$
2 \cos \left(2 \pi v / m^{\prime}\right)=\frac{2 \sin \left(4 \pi v / m^{\prime}\right)}{2 \sin \left(2 \pi v / m^{\prime}\right)},
$$


the product of cosines must also equal 1 . Now from (35),

$$
\begin{aligned}
c_{\phi(m) / 2} & =\prod_{v=1,\left(v, m^{\prime}\right)=1}^{[m / 2]} i^{*} \sqrt{l}\left(\frac{v}{l}\right)\left(\zeta_{m}^{v}+(-1)^{(l-1) / 2} \zeta_{m}^{-v}\right) \\
& =\left((-1)^{(l-1) / 2} l\right)^{\phi(m) / 4} \prod_{v=1,\left(v, m^{\prime}\right)=1}^{\left[m^{\prime} / 2\right]}\left(\frac{v}{l}\right)\left(\zeta_{m^{\prime}}^{v}+(-1)^{(l-1) / 2} \zeta_{m^{\prime}}^{-v}\right)
\end{aligned}
$$

or just

$$
\left((-1)^{(l-1) / 2} l\right)^{\phi(m) / 4}(-1)^{N}(-1)^{\frac{l-1}{2} \frac{\phi\left(m^{\prime}\right)}{4}}=(-1)^{N} l^{\phi(m) / 4}
$$

by (36), where $N$ counts the number of reduced residues $v$ modulo $m^{\prime}$ in the interval $\left(0, m^{\prime} / 2\right)$ with $\left(\frac{v}{l}\right)=-1$. This completes the proof of the proposition.

Before concluding this section I wish to remark that one obtains a variant for the sums $S_{n}$ in (26) when $n$ is even using the fact that $P(X)=P_{m, l}(X)$. $P_{m, l}(-X)$, so that $S_{n}=\frac{1}{2} S_{n}^{\prime}$, where $S_{n}^{\prime}$ is the $n$th power sum associated to $P(X)$ with $n$ even. Now from (28),

$$
\begin{aligned}
\log P(X)= & \phi(m) \log E_{l}(X)+\sum_{d \mid m^{\prime}} \mu(d) \log \left(1-(-1)^{(l-1) / 2} A_{l}(X)^{2 m / m^{\prime}}\right) \\
= & -\phi(m) \sum_{n=1}^{\infty}\left(\begin{array}{c}
2 n \\
n
\end{array}\right) \frac{l^{n} X^{2 n}}{2 n} \\
& -\sum_{d \mid m^{\prime}} \mu(d) \sum_{v=1}^{\infty} \frac{(-1)^{(l-1) v / 2}}{v} A_{l}(X)^{2 m v / m^{\prime}} .
\end{aligned}
$$

Thus if $n$ is even,

$$
S_{n}=l^{n / 2} \frac{\phi(m)}{2}\left(\begin{array}{c}
n \\
n / 2
\end{array}\right)+l^{n / 2} \sum_{d \mid m} \mu(d) \frac{m}{d} \sum_{t=1}^{[n d / 2 m]}(-1)^{(l-1) t / 2}\left(\begin{array}{c}
n \\
n / 2-m t / d
\end{array}\right)
$$

as an alternative expression for $S_{n}$ in (26).

\section{References}

[1] B. C. Berndt, R. J. Evans and K. S. Williams, Gauss and Jacobi Sums, WileyInterscience, New York, 1998.

[2] Z. I. Borevich and I. R. Shafarevich, Number Theory, Academic Press, New York, 1966.

[3] R. P. Brent, On computing factors of cyclotomic polynomials, Math. Comp. 61 (1993), 131-149.

[4] C. F. Gauss, Disquisitiones Arithmeticae, Yale Univ. Press, New Haven, CT, 1966. 
[5] S. Gupta and D. Zagier, On the coefficients of the minimal polynomial of Gaussian periods, Math. Comp. 60 (1993), 385-398.

[6] S. Gurak, Minimal polynomials for circular numbers, Pacific J. Math. 112 (1984), 313-331.

[7] -, Minimal polynomials for Gauss circulants and cyclotomic units, ibid. 102 (1982), 347-353.

[8] H. Hasse, Vorlesungen über Zahlentheorie, Springer, Berlin, 1950.

[9] E. Lucas, Théorèmes d'arithmétique, Atti Roy. Acad. Sci. Torino 13 (1877-78), 271-284.

[10] J. Neukirch, Class Field Theory, Springer, New York, 1986.

[11] T. J. Rivlin, Chebyshev Polynomials From Approximation Theory to Algebra and Number Theory, Wiley, New York, 1990.

[12] H. Salié, Über die Kloostermanschen Summen $S(u, v ; q)$, Math Z. 34 (1932), 91-109.

[13] A. Schinzel, On primitive prime factors of $a^{n}-b^{n}$, Proc. Cambridge Philos. Soc. 58 (1962), 555-562.

[14] P. Stevenhagen, On Aurifeuillian factorizations, Nederl. Akad. Wetensch. Indag. Math. 49 (1987), 451-468.

[15] L. C. Washington, Introduction to Cyclotomic Fields, Grad. Texts in Math. 83, Springer, New York, 1982.

[16] W. Watkins and J. Zeitlin, The minimal polynomial of $\cos (2 \pi / n)$, Amer. Math. Monthly 100 (1993), 471-474.

University of San Diego

San Diego, CA 92110, U.S.A.

E-mail: gurak@sandiego.edu

Received on 13.6.2005

and in revised form on 4.11.2005 